\title{
Cils et migrations neuronales
}

\section{Christine Métin}

> Dans un article fondateur paru en 1977, G. Albrecht-Buehler décrivait un cil primaire à la surface de fibroblastes en migration, et remarquait que le cil est orienté parallèlement à la direction de migration des cellules. La présence d'un cil primaire a été notée sur les progéniteurs neuraux et sur les neurones postmitotiques depuis longtemps. Cependant, ce n'est que récemment qu'un cil primaire a été observé sur les interneurones corticaux en migration. Comme dans les fibroblastes, le cil des interneurones contrôle la direction de la migration. Il joue un rôle particulièrement important dans la réorientation des interneurones vers la plaque corticale. Le morphogène Shh (Sonic hedgehog), qui est exprimé dans les voies de migration des interneurones, est l'un des signaux qui contrôlent cette réorientation. <

Les neurones naissent et se multiplient au centre du cerveau dans le neuroépithélium bordant les ventricules, puis migrent vers les régions périphériques où ils s'organisent en noyaux ou en couches. Les propriétés fonctionnelles des structures ainsi formées dépendent, non seulement des caractéristiques fonctionnelles des neurones qui les composent, mais aussi de leur organisation spatiale. Les propriétés migratoires des neurones influencent directement leur arrangement final. Les anomalies de migration qui surviennent pendant le développement du cortex cérébral sont responsables de défauts fonctionnels graves, comme l'épilepsie et le déficit intellectuel ; ces signes cliniques sont aussi retrouvés dans les ciliopathies. Le cil primaire que nous avons observé sur les futurs neurones inhibiteurs du cortex cérébral chez l'embryon de souris est un acteur important du développement cortical, car il régule la migration et le positionnement ultérieur de ces neurones dans le cortex [1, 2].

Cet article fait partie du numéro thématique de médecine/sciences intitulé «Cils primaires et ciliopathies $\gg\left(\mathrm{m} / \mathrm{s} \mathrm{n}^{\circ} 11\right.$, vol. 30, novembre 2014).

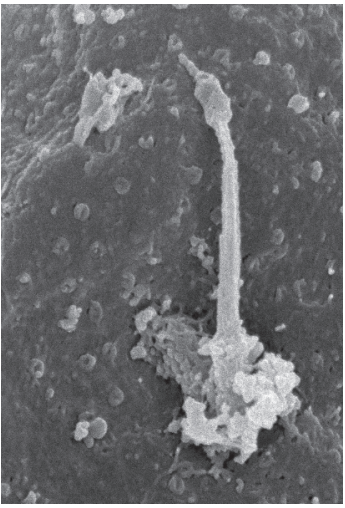

Institut du Fer à moulin, Inserm UMRS 839, université Pierre et Marie Curie, 17, rue du Fer à Moulin, 75005 Paris, France. christine.metin@inserm.fr

\section{Les mouvements migratoires dans le cortex cérébral embryonnaire}

Le cortex cérébral comprend essentiellement deux populations de neurones: les neurones excitateurs et les interneurones inhibiteurs. Les neurones excitateurs sont organisés en couches parallèles à la surface du cortex. Ils sont produits localement et rejoignent la plaque corticale qui préfigure le cortex adulte (Figure IA, zone orange) en migrant le long des cellules de glie radiaire, leurs cellules progénitrices. On sait depuis une vingtaine d'années que les interneurones inhibiteurs GABA (acide $\gamma$-aminobutyrique)-ergiques sont générés en dehors du cortex, dans la partie ventrale du cerveau antérieur [3] (Figure 1A). Ils naissent en majorité dans l'éminence ganglionnaire médiane ( $(G M)$, une structure qui s'efface chez l'embryon à la fin de la gestation, et dans deux autres régions identifiées plus récemment [4]. Ces trois régions produisent différentes classes d'interneurones qui migrent d'abord vers la frontière séparant le cerveau ventral et le cortex. Après avoir traversé cette frontière, les neurones se distribuent de part et d'autre de la plaque corticale et s'organisent en deux flux parallèles (Figure 1A). Un flux dense occupe la zone marginale au-dessus de la plaque corticale. Un second flux, assez large, formé d'une cohorte de cellules migrant individuellement, s'établit le long de la zone proliférative. Régulièrement, des cellules quittent ces flux tangentiels et prennent une direction oblique ou radiale pour rejoindre et coloniser la plaque corticale sous laquelle se forme un flux supplémentaire. Les changements de trajectoire affectent à chaque instant un nombre limité de cellules de la cohorte, à tous les niveaux latéro-médians. Ces réorientations sont compatibles avec un processus stochastique, comme avec un mécanisme de sortie des flux tangentiels finement régulé.

\section{Transformations accompagnant la migration des neurones GABAergiques}

Pour se déplacer, les neurones GABAergiques embryonnaires étendent à l'avant un prolongement explorateur branché [5-7] (Figure 1B). 
A Voies de migration des futurs interneurones dans le cortex embryonnaire

Souris $\varepsilon 13,5$
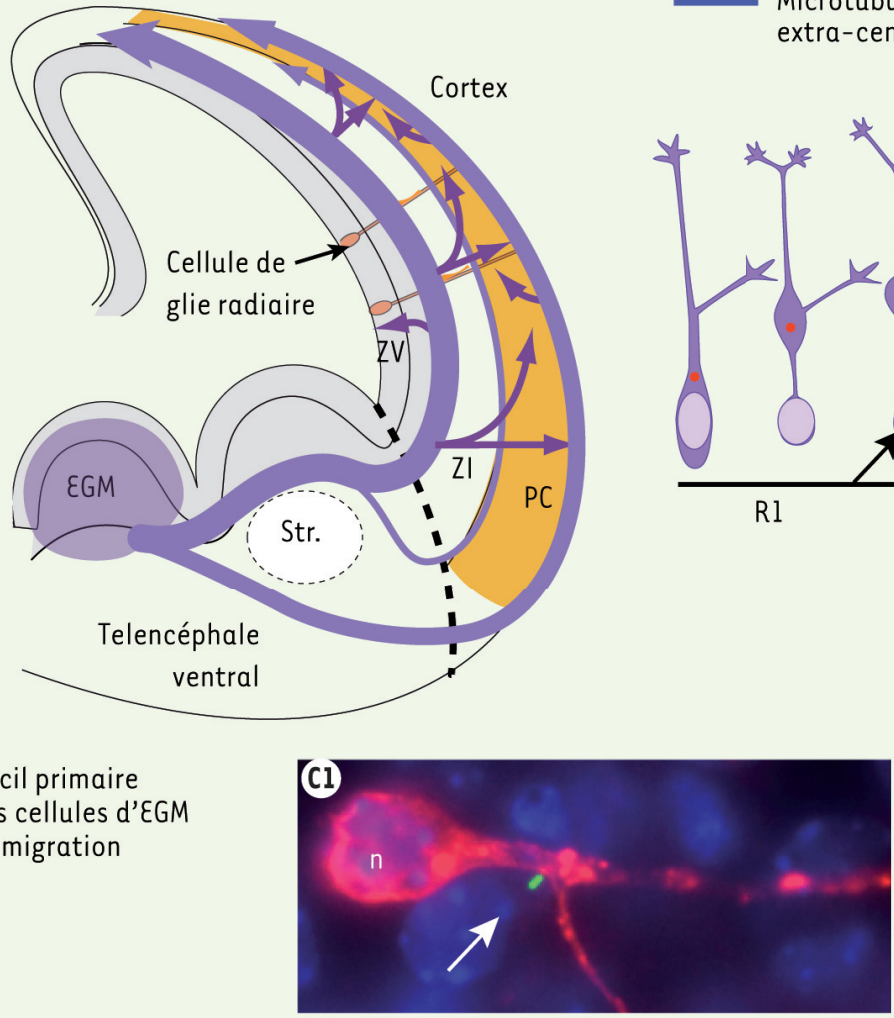

C Le cil primaire des cellules d'EGM en migration
B Cycle de migration des cellules d'EGM et organisation des microtubules

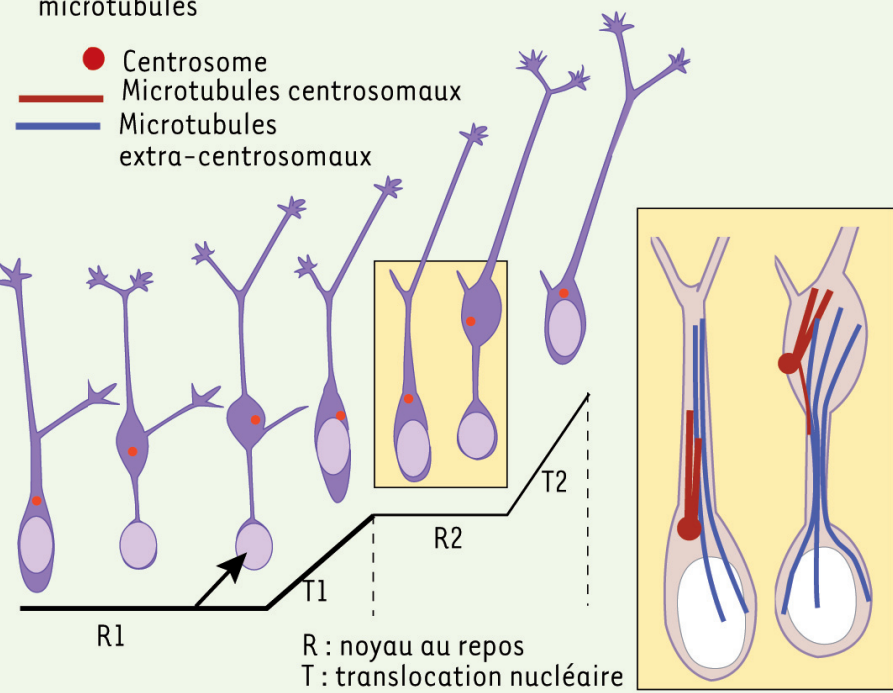

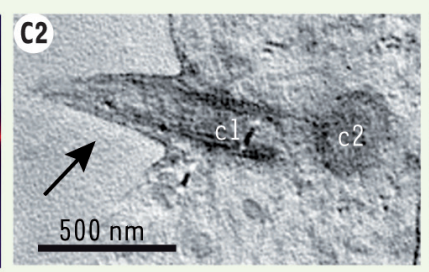

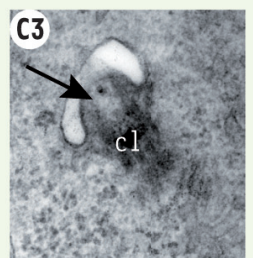

Figure 1. Les interneurones corticaux nés dans l'EGM assemblent un cil primaire pendant leur phase de migration. A. Voies de migration des interneurones dans le cortex (flèches violettes). B. Cycle de migration en deux phases des interneurones corticaux. Le schéma représente deux cycles de migration, R1-T1 et R2-T2 : (1) le centrosome associé à un réseau de microtubules (rouge) migre vers l'avant de la cellule pendant la phase de repos du noyau (R); (2) le noyau migre vers le centrosome (T). C. Un cil primaire souvent court (flèches blanches en $\mathbf{C l}$ et noires en $\mathbf{C 2 - 3}$ ) prolonge le corps basal ( $\mathrm{cl}$ ) qui est, soit ancré à la membrane plasmique (C2), soit associé à une vésicule ciliaire cytoplasmique (C3). EGM: éminence ganglionnaire médiane ; PC : plaque corticale ; Str. : striatum ; ZI : zone intermédiaire ; ZV : zone ventriculaire ; 2 dans le panneau C : centriole fils orienté perpendiculairement au corps basal.

À chaque bifurcation, une branche est sélectionnée pour la progression des organites cytoplasmiques. L'orientation des branches préfigure la trajectoire cellulaire, et les changements de direction (notamment vers la plaque corticale) nécessitent la formation de branches divergentes par rapport à la direction de migration initiale [5]. Le processus de formation et d'élongation des branches n'est pas continu, mais corrélé aux mouvements nucléaires saltatoires [6]. L'arborisation neuritique se développe pendant la phase de repos du noyau (Figure IB, R), alors que les autres organites cytoplasmiques migrent en avant. Ils migrent parfois si loin du noyau qu'un prolongement membranaire très fin relie le compartiment nucléaire resté à l'arrière et le renflement avant qui contient le centrosome. Puis, le noyau rejoint le centrosome; c'est la seconde phase du cycle de migration (Figure $1 B, T$ ), souvent brève et généralement concomitante de la rétraction de l'arrière du corps cellulaire [8].
L'ampleur des déformations subies par le corps cellulaire des neurones GABAergiques migrateurs nous a conduits à examiner en détail l'organisation de leurs microtubules. Nos études en tomographie électronique ont montré que les neurones GABA renferment un réseau de microtubules attachés au centrosome et un réseau de microtubules qui ne sont pas ancrés au centrosome $[1,9]$ (Figure 1B). Ces derniers pourraient guider les mouvements du noyau vers le renflement contenant le centrosome. Le groupe de Kengaku [10] avait déjà proposé un modèle d'organisation des microtubules comparable dans les neurones en grain du cervelet. Notre modèle, comme celui de Kengaku, diffère significativement du modèle classique d'organisation des 
microtubules ${ }^{1}$ dans les neurones migrateurs [11]. II rend compte d'une propriété inattendue du centrosome, qui est la capacité du centriole père à s'attacher à la membrane plasmique par un cil primaire (Figure IC). Cet ancrage membranaire est plus fréquemment observé quand le centrosome est localisé loin devant le noyau. Des faisceaux de microtubules sont alors attachés par des appendices latéraux au centriole père, devenu un corps basal ${ }^{2}[12](\rightarrow)$.

$\rightarrow$ Voir la Synthèse de A. Benmerah, page 962 de ce numéro Lorsque le centrosome est localisé près du noyau, dans le cytoplasme (Figure 1C), une grande vésicule est fréquemment associée au pôle distal du centriole père. Elle peut contenir un cil rudimentaire. Cette dernière configuration rappelle les stades précoces de la ciliogenèse décrits il y a plus de 50 ans par Sorokin [13] dans les fibroblastes et les cellules musculaires en différenciation. À la lumière de ces observations, nous proposons que différents stades de la ciliogenèse soient associés à différentes phases du cycle de migration. D'autres hypothèses sont envisageables; par exemple que certains stades de différenciation des neurones GABAergiques soient corrélés à des phases particulières de la ciliogenèse.

\section{Quelle(s) fonction(s) pour le cil primaire des neurones migrateurs GABAergiques du cortex ?}

Le rôle du cil primaire dans la migration cellulaire a été étudié principalement dans des cellules adhérentes [14] (fibroblastes, cellules de muscle lisse). En 1977, Albrecht Buehler a décrit pour la première fois, à la surface des fibroblastes en migration, un cil primaire orienté parallèlement à la direction de migration [15]. Dans ces modèles cellulaires, le cil contrôle la direction de la migration. II semble fonctionner comme une antenne sensorielle capable de collecter et de relayer des signaux extracellulaires vers le centrosome et les microtubules [16].

Nous avons recherché si le cil est impliqué dans le contrôle de la polarité des neurones GABAergiques migrateurs. Pour répondre à cette question, nous avons invalidé la sous-unité KIF3A de la kinésine 2 qui contrôle la construction du cil, spécifiquement dans les neurones GABAergiques issus de I'EGM. Le défaut le plus évident des neurones GABAergiques mutants cultivés sur un substrat isotrope (c'est-à-dire présentant les mêmes propriétés sur toute sa surface) de cellules corticales sauvages dissociées est une stabilisation des prolongements de migration. Celle-ci semble résulter d'un changement des propriétés dynamiques du cytosquelette de microtubules et de l'adhésion cellulaire. Dans un environnement structuré et polarisé, tel que le cortex embryonnaire, les neurones GABAergiques mutants forment des flux de migration tangentiels beaucoup plus denses que les neurones sauvages, et colonisent la plaque corticale avec retard (Figure 2A-B). Leurs prolongements sont en majorité orientés tangentiellement, et très peu se réorientent vers la plaque corticale. L'invalidation

${ }^{1}$ Selon ce modèle, tous les microtubules sont attachés au centrosome et les microtubules dirigés vers

l'arrière de la cellule forment une cage autour du noyau.

${ }^{2}$ Le corps basal est associé à la base du cil primaire et dérive du centriole père. du cil primaire altère donc la capacité des neurones GABAergiques à réorienter leur trajectoire (branches neuritiques) pour quitter le flux de migration tangentiel [1]. Ce défaut ressemble à celui des fibroblastes invalidés pour le cil primaire, qui perdent la capacité de se réorienter vers une zone dépourvue de cellules et de la coloniser [16]. Les neurones GABAergiques mutants pour ARL13b (ADP-ribosylation factor-like 13b), une GTPase adressée à la membrane ciliaire, présentent, en plus des défauts de migration décrits ci-dessus, un fort ralentissement de la migration [2]. Cet effet du cil sur le contrôle de la dynamique de migration n'est pas observé dans les mutants que nous avons étudiés, et n'a pas été décrit dans les fibroblastes. II est possible que les fonctions extraciliaires d'ARL13b contribuent également aux défauts de migration du mutant.

\section{Contrôle de la migration des neurones GABAergiques par les signaux Shh}

Le cil primaire des vertébrés est nécessaire à l'activation de la voie patched-smoothened (Ptc-Smo) par les signaux Hedgehog, en particulier par Shh (Sonic hedgehog) [17], qui est un morphogène exprimé d'abord dans le cerveau ventral dont il contrôle les étapes précoces du développement, puis dans le télencéphale

$(\rightarrow)$ Voir la Synthèse de C. Laclef, page 980 dorsal [18] $(\rightarrow)$.

Shh est exprimé dans les voies de migration tangentielle du cortex au stade embryonnaire, lorsque les cellules de l'EGM colonisent le cortex [19] (Figure 2D). Nous avons donc recherché si la migration des neurones GABAergiques est influencée par Shh, et si l'ablation génétique du cil primaire perturbe la réponse des neurones GABAergiques à Shh.

L'application de cyclopamine, un inhibiteur de la voie Ptc-Smo, sur des tranches de cortex (Figure 2C) mime l'effet de l'ablation génétique du cil primaire sur la distribution corticale des cellules d'EGM (Figure 2B). À l'inverse, les agonistes de la voie de signalisation, SAG (Smo agonist) et Shh, favorisent la dispersion des cellules d'EGM en dehors de leur voie de migration tangentielle. Cette réponse est partiellement abolie par l'ablation génétique du cil primaire. L'activité du cil pourrait donc favoriser la colonisation progressive du cortex cérébral par les neurones GABAergiques. En effet, des signaux attractifs maintiennent les neurones GABA dans les flux de migration tangentiels, en particulier la chimiokine CXCL12 et la neuréguline 1 [4]. L'exposition au signal Shh pourrait permettre à certains neurones GABAergiques de quitter la voie de migration tangentielle où ils sont retenus par ces signaux attractifs (Figure $2 \varepsilon$ ). 

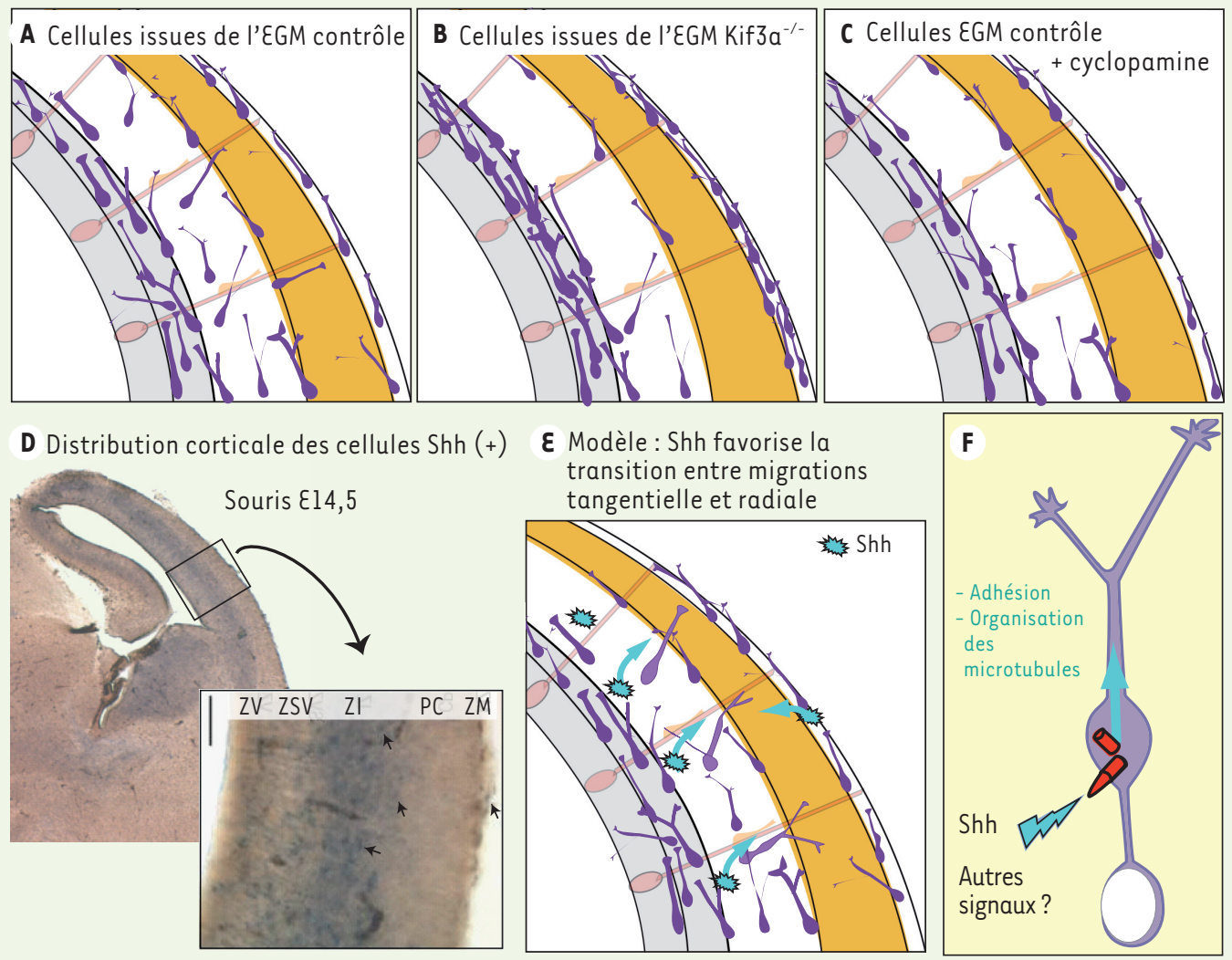

$\boldsymbol{\varepsilon}$ Modèle : Shh favorise la transition entre migrations tangentielle et radiale
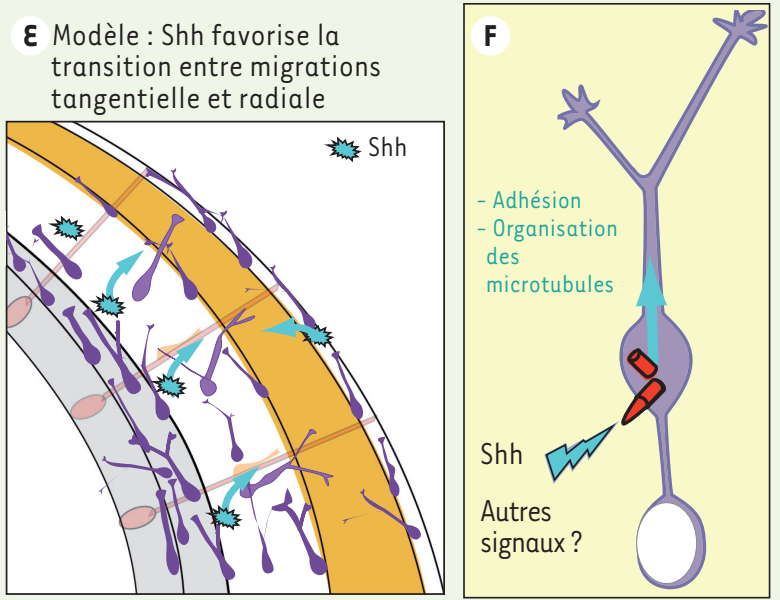

Figure 2. Le cil primaire des interneurones collecte des signaux contrôlant la migration. Les interneurones colonisent la plaque corticale (en orange, A) à partir des flux de migration tangentiels profonds et superficiels. L'ablation génétique du cil primaire (B) et l'inhibition pharmacologique de la voie de signalisation Sonic hedgehog-patched-smoothened (Shh-Ptc-Smo) (C) maintiennent les interneurones dans les voies tangentielles. Shh, qui est exprimé au voisinage des interneurones en migration $(D)$, favorise leur réorientation vers la plaque corticale $(\varepsilon)$. Les signaux collectés par le cil primaire influencent les propriétés migratoires des interneurones corticaux, en particulier l'organisation des microtubules et les propriétés d'adhérence (F). ZSV : zone sous-ventriculaire ; ZM : zone médiane.

L'ensemble de ces résultats identifient le cil primaire comme un nouveau site de transduction des signaux impliqués dans le contrôle de la migration des interneurones GABAergiques (Figure 2F). Toutefois, ni les mécanismes subcellulaires qui contrôlent l'organisation des microtubules et l'adhésion, ni le mécanisme d'action de Shh, ne sont compris. Ces premières observations soulèvent de nombreuses autres questions. Par exemple, nous ne savons pas si tous les neurones GABAergiques, ou seulement certaines sous-classes, assemblent un cil primaire. Nous ne savons pas non plus si d'autres types de neurones migrateurs ont un cil. Des hétérotopies observées dans le cortex de plusieurs mutants ciliaires [20] suggèrent que le cil pourrait aussi contrôler la migration radiale, même si un cil primaire fonctionnel n'a pas encore été décrit sur les neurones corticaux embryonnaires à migration radiale. Nous proposons que la ciliogenèse soit corrélée au cycle de migration, mais le comportement dynamique du cil pendant le cycle de migration reste à caractériser. La recherche des réponses à ces nombreuses questions devrait nous conduire à identifier de nouveaux processus cellulaires essentiels à la construction du cortex cérébral, et possible- ment responsables des anomalies cognitives associées à certaines ciliopathies $[18,21](\rightarrow)$.

\section{SUMMARY}

Cilia and neuronal migrations

In a landmark paper published in 1977, G. AlbrechtBuehler described a primary cilium on the surface of migrating fibroblasts, and noticed that cilia are oriented parallel to the direction of migration of fibroblasts. While the presence of a primary cilium on neural progenitors and on post-mitotic neurons was noted long ago, it has been observed on migrating cortical interneurons only recently. As in fibroblasts, the cilium of interneurons controls the directionality of migration. It plays an important role in the reorientation of cortical interneurons towards the cortical plate. The morphogen Shh, which is expressed in the migratory pathway of interneurons, is one of the signals that control this reorientation. $\diamond$ 


\section{LIENS D'INTÉRÊT}

L'auteur déclare n'avoir aucun lien d'intérêt concernant les données publiées dans cet article.

\section{RÉFÉRENCES}

1. Baudoin JP, Viou L, Launay PS, et al. Tangentially migrating neurons assemble a primary cilium that promotes their reorientation to the cortical plate. Neuron $2012 ; 76: 1108-22$

2. Higginbotham $\mathrm{H}$, Eom TY, Mariani LE, et al. Arl $13 \mathrm{~b}$ in primary cilia regulates the migration and placement of interneurons in the developing cerebral cortex. Dev Cell 2012;23:925-38.

3. Marin 0 , Rubenstein JL. A long, remarkable journey: tangential migration in the telencephalon. Nat Rev Neurosci $2001 ; 2: 780-90$.

4. Marin 0 . Cellular and molecular mechanisms controlling the migration of neocortical interneurons. Eur J Neurosci $2013 ; 38: 2019-29$.

5. Polleux F, Whitford KL, Dijkhuizen PA, et al. Control of cortical interneuron migration by neurotrophins and PI3-kinase signaling. Development $2002 ; 129$ : 3147-60.

6. Bellion A, Baudoin JP, Alvarez C, et al. Nucleokinesis in tangentially migrating neurons comprises two alternating phases: Forward migration of the Golgi/centrosome associated with centrosome splitting and myosin contraction at the rear. J Neurosci $2005 ; 25: 5691-9$.

7. Martini FJ, Valiente M, Lopez-Bendito G, et al. Biased selection of leading process branches mediates chemotaxis during tangential neuronal migration. Development $2008 ; 136: 41-50$

8. Baffet $A$. La migration nucléaire dans les progéniteurs neuronaux. Quand le cerveau joue au yo-yo. Med Sci (Paris) $2014 ; 30: 30-2$.

9. Pilon A, Poüs C. Compartimentation et plasticité du réseau microtubulaire. Med Sci (Paris) 2013 ; $29: 194-9$.

10. Umeshima H, Hirano T, Kengaku M. Microtubule-based nuclear movement occurs independently of centrosome positioning in migrating neurons. Proc Natl Acad Sci 20007 ; 104 : 16182-7.

11. Tsai LH, Gleeson JG. Nucleokinesis in neuronal migration. Neuron $2005 ; 46: 383-8$.
12. Benmerah A. La poche ciliaire : fruit des liaisons du centrosome avec le trafic vésiculaire. Med Sci (Paris) 2014 ; 30 : 962-7.

13. Sorokin S. Centrioles and the formation of rudimentary cilia by fibroblasts and smooth muscle cells. J Cell Biol $1962 ; 15: 363-73$.

14. Christensen ST, Veland IR, Schwab A, et al. Analysis of primary cilia in directional cell migration in fibroblasts. Methods Enzymol 2013 ; 525 : $45-58$.

15. Albrecht-Buehler G. Phagokinetic tracks of $3 T 3$ cells: Parallels between the orientation of track segments and of cellular structures which contain actin or tubulin. Cell $1977 ; 12: 333-9$.

16. Schneider L, Cammer L, Lehman J, et al. Directional cell migration and chemotaxis in wound healing response to PDGF-AA are coordinated by the primary cilium in fibroblasts. Cell Physiol Biochem $2010 ; 25: 279-92$.

17. Ruat $M$, Angot $\varepsilon$, Traiffort $\varepsilon$. Sonic Hedgehog, un morphogène en quête de fonction dans le cerveau adulte. Med Sci (Paris) $2011 ; 27: 979-85$.

18. Laclef $\mathrm{C}$. Le cil primaire, orchestrateur de la morphologie cérébrale. Med Sci (Paris) $2014 ; 30: 980-90$.

19. Komada M, Saitsu H, Kinboshi M, et al. Hedgehog signaling is involved in development of the neocortex. Development 2008 ; $135: 2717-27$.

20. Willaredt MA, Tasouri $\varepsilon$, Tucker KL. Primary cilia and forebrain development. Mech Dev $2012 ; 130: 373-80$.

21. Bachmann-Gagescu R. Complexité génétique des ciliopathies et identification de nouveaux gènes. Med Sci (Paris) 2014 ; 30 : 1011-23.

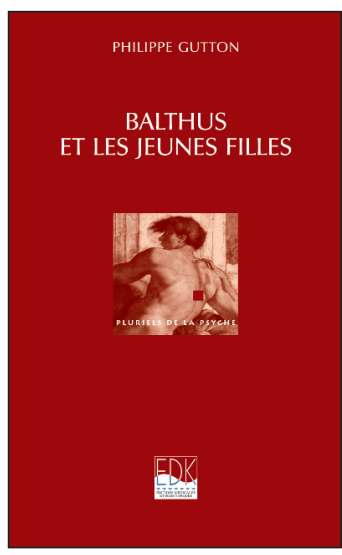

ISBN : 978-2-8425-4190-3 204 pages
À n’en pas douter Balthus (1908-2001) aura été l'un des peintres les plus singuliers du XXe siècle. Les décors étranges où évoluent ses personnages l’ont longtemps inscrit dans la mouvance surréaliste, influence pourtant qu'il rejetait. Les poses suggestives de ses modèles, jeunes filles en fleur, adolescentes à peine pubères, ont incité maints commentateurs à le considérer comme un peintre érotique. Mais là encore Balthus réfutait pareille définition réductrice de son art, lui qui se revendiquant fervent catholique, affirmait: «Le peintre doit être religieux ou n'être pas. »

Philippe Gutton, psychanalyste spécialiste de l'adolescence, reprend la question à ses racines dans un souci de ne pas réduire à une interprétation simplificatrice une création si originale. Quelle énigme recèle cette œuvre ? Que nous disent les figures troublantes qui la hantent? Que révèlent celles-ci de ce temps charnière qu'est l'adolescence? Qu'en tant qu'âge de découverte de la génitalité et de la mort, elle est paradigmatique de la créativité à laquelle tout être humain est tenu de croire s'il veut échapper à la mélancolie; qu'elle ouvre au mystère de la femme advenante, au secret du vivant, bref qu'interrogeant la puberté féminine en tant qu'elle symbolise l'origine, Balthus, selon la meilleure esthétique, tente et réussit à rendre visible l'invisible de l'humain se faisant.

Philippe GUTTON est psychiatre, psychanalyste. Professeur honoraire des universités, il a fondé en 1983 et dirige depuis la revue Adolescence. Il est l'auteur de nombreux ouvrages, dont, Le pubertaire, Paris, PUF, 1991 ; Violence et adolescence, Paris, In Press, 2002 ; Le génie adolescent, Paris, Odile Jacob, 2008 ; La chambre des amants, Paris, Odile Jacob, 2011.

UU À retourner à EDK, 109, avenue Aristide Briand, 92541 Montrouge Cedex - Tél. : 0141177405 - Fax : 0149850345 - E-mail : edk@edk.fr

2 NOM : Prénom :

Adresse :

Code postal :

Ville :

Pays :

Fonction :

Je souhaite recevoir l'ouvrage Balthus et les jeunes filles : $20 €+3 €$ de port $=\mathbf{2 3} €$ TTC

en ................. exemplaire, soit un total de ....................................... €

UU Par chèque, à l'ordre de $\mathbf{E} \mathbf{D} \mathbf{K}$

$\square$ Par carte bancaire : $\square$ Visa $\square$ Eurocard/Mastercard

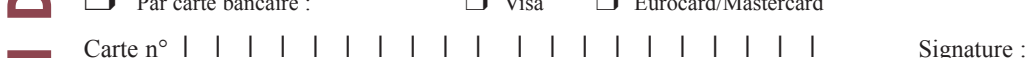

Date d'expiration: $\quad 11|1|$

$\mathrm{N}^{\circ}$ de contrôle au dos de la carte : 